H. Drechsel, J. Rahe, W. Wargau

Remeis-Sternwarte Bamberg, Astronomical Institute University Erlangen-Nürnberg / F.R.G.

B. Wolf

Landessternwarte Heidelberg / F.R.G.

\title{
ABSTRACT
}

Optical photometric and spectroscopic observations of the eclipsing binary SV Cen were used to derive orbital elements and absolute dimensions of the peculiar early-type contact system. UV spectroscopic observations obtained with the IUE satellite yielded the detection of mass loss from the system forming an expanding circumbinary envelope. $S V$ Cen exhibits a non-uniform rate of period decrease, with major variations on a time scale of a few years. The period changes are explained in terms of loss of angular momentum carried away by the ejected matter. Short-term fluctuations of the stellar wind on a time scale of a few hours were found from an analysis of the envelope line profiles of UV resonance lines. A model of the system is derived, and the evolutionary stage and interaction processes are discussed.

\section{A. OBSERVATIONS}

Information about the observational material is presented in tahle 1. We have obtained ootical photoelectric measurements between 1978 and 1981 to serve two purposes: first, to derive orbital elements from an analysis of the UBV light curves; and second, to obtain accurate times of minimum epochs in order to determine the variation and amount of period decrease.

Further, we took a total of 30 coudé spectrograms between 1977 and 1979, in order to investigate the optical spectral range and to derive the mass ratio.

Finally, we obtained eight UV spectra during two shifts with the IUE satellite in 1979 and 1981, which cover the short and long wavelength ranges in both high and low reso- 
lution. The UV spectroscopy yielded the detection of mass loss from the system, and proved very useful for the investigation of properties of the expanding envelope and for the analysis of interaction processes of the binary components.

Table 1. Optical and UV Observations of SV Cen

\section{Optical Photometry}

\begin{tabular}{l|l|l} 
Dates & Instrument & Remarks \\
\hline 1978, Apri1 2-7 & ESO 50cm & UBV light curves \\
1979, May 25,28,30 & ESO BOchum 61 cm & light curves, minima \\
1980, Feb.29, March 3 & ESO 50cm & minima \\
1981, March 21,31 & ESO 50cm & minima \\
\hline
\end{tabular}

\section{Optical spectroscopy}

30 coudé spectrograms, between July 1977 and May 1979;

dispersion: $20 \AA / \mathrm{mm}$; emulsion: Kodak IIaO (B); instrument: ESO $1.52 \mathrm{~m}$ telescope

\section{UV Spectroscopy}

\begin{tabular}{l|l|l} 
Dates & IUE Image Nos. & Resolution \\
\hline 1979, Nov. 3.576 & LWR 6014 & low \\
1979, Nov. 3.580 & SWP 7078 & low \\
1979, Nov. 3.631 & LWR 6015 & high \\
1979, Nov. 3.695 & SWP 7079 & high \\
1981, Jan. 12.407 & SWP 11036 & low \\
1981, Jan. 12.435 & LWR 9697 & high \\
1981, Jan. 12.525 & SWP 11037 & high \\
1981, Jan. 12.596 & LWR 9698 & low \\
\hline
\end{tabular}

\section{B. ORBITAL ELEMENTS AND ABSOLUTE DIMENSIONS}

Orbital elements and absolute dimensions of SV Cen were derived from the optical photometric and spectroscopic data. The UBV light curves were analyzed with the light curve and differential corrections procedure of Wilson and Devinney. The spectroscopic mass ratio $(q=1.25)$ and the primary effective temperature $\left(T_{1}=23000 \mathrm{~K}\right)$ were used as fixed parameters, and model assumptions for limb and gravity darkening and for the color-dependent albedos had to be applied. Satisfactory convergence was found in the contact configuration mode, yielding the parameters listed in table 2. 
Table 2. Orbital Elements and Absolute Dimensions of SV Cen

\begin{tabular}{|c|c|c|c|}
\hline $\begin{array}{l}q \\
i \\
p\end{array}$ & $\begin{array}{l}=M_{2} / M_{1}=1.25 \\
=81.8 \pm 0: 1 \\
=1.6585 \text { (decreasing) }\end{array}$ & $\begin{array}{l}L_{1}(V) / L_{1}+L_{2} \\
L_{1}(B) / L_{1}+L_{2} \\
L_{1}(U) / L_{1}+L_{2}\end{array}$ & $\begin{array}{ll}= & 0.60 \pm 0.01 \\
= & 0.64 \pm 0.01 \\
= & 0.65 \pm 0.01\end{array}$ \\
\hline $\begin{array}{l}\mathrm{Sp}_{1} \\
\mathrm{Sp}_{2}\end{array}$ & $\begin{array}{l}=\mathrm{B} 1 \mathrm{~V} \\
=\mathrm{B} 6.5 \mathrm{III}\end{array}$ & $\begin{array}{l}L_{1} \text { (bolo) } \\
L_{2}(\text { bolo) }\end{array}$ & $\begin{array}{l}=11700 \mathrm{~L}_{\odot} \\
=1900 \mathrm{~L}_{\odot}\end{array}$ \\
\hline $\begin{array}{l}\mathrm{T}_{1} \\
\mathrm{~T}_{2}\end{array}$ & $\begin{array}{l}=23000 \mathrm{~K} \\
=14000 \mathrm{~K}\end{array}$ & $\begin{array}{l}M_{1}(V) \\
M_{2}(V)\end{array}$ & $\begin{array}{l}=-3 m_{1} \\
=\quad-2 \dot{m}_{3}\end{array}$ \\
\hline $\begin{array}{l}a_{1} \\
a_{2}\end{array}$ & $\begin{array}{l}=\quad 8.5 R_{\odot} \\
=\quad 6.8 R_{\odot} \\
=15.3 R_{\odot}\end{array}$ & $\begin{array}{l}\Omega_{1}=\Omega_{2} \\
\Omega_{\text {ic }} \\
\Omega_{\text {OC }}\end{array}$ & $\begin{array}{l}=3.611 \pm 0.01 \\
=4.13 \\
=3.57\end{array}$ \\
\hline $\begin{array}{l}M_{1} \\
M_{2}\end{array}$ & $\begin{array}{l}=\quad 7.7 \mathrm{M}_{\odot} \\
=\quad 9.6 \mathrm{M}_{\odot}\end{array}$ & $\begin{array}{l}E(B-V) \\
A_{V}\end{array}$ & $\begin{array}{l}=\quad \mathrm{m} 27 \\
=\quad \mathrm{m} \cdot 9\end{array}$ \\
\hline $\mathrm{R}_{1}$ & $\begin{array}{l}=6.8 \mathrm{R}_{\odot} \\
=7.4 \mathrm{R}_{\odot}\end{array}$ & $\mathrm{d}$ & $=1800 \mathrm{pc}$ \\
\hline
\end{tabular}

The less massive component of spectral type B1 is the photometric primary. With a mass of about 8 and a radius of about 7 solar units, it is located slightly above the main sequence. The secondary is of spectral type B6.5, and has similar parameters. The system is in overcontact, with both stars overfilling their Roche lobes. The separation of the mass centers amounts to only about 15 solar units.

\section{B. PRESENCE OF AN EXPANDING ENVELOPE}

The period of 1.6 days is rapidly decreasing. SV Cen has previously been regarded as a rare example of a binary being presently observed during the early part of mass exchange, prior to reversal of mass ratio. In order to explain the period decrease, it was assumed that the mass is flowing from the more massive to the less massive component. Since no indication for circumstellar material was found in the optical spectral range, the mass transfer was thought to be fully conservative and to take place within the common contact surface (Wilson and starr, 1976).

Our UV spectra, however, clearly show the presence of an expanding circumbinary envelope (Drechsel et al., 1980). Apart from photospheric and in some cases interstellar contributions, the most prominent features in the high resolution IUE spectra are highly displaced envelope components of reso- 
nance lines of C II, Si IV, C IV, AI II,III, and Mg II, as well as of the metastable Fe III multiplets 34 and 48 . The central wavelengths of the envelope lines are shifted by -700 to $-1200 \mathrm{~km} \mathrm{~s}^{-1}$, and the velocities of the shortward edges are ranging from -900 to $-2000 \mathrm{~km} \mathrm{~s}^{-1}$. A comparison with the escape velocity of the order of $750 \mathrm{~km} \mathrm{~s} \mathrm{~s}^{-1}$ obviously suggests the occurrence of mass loss from the system. Radiation pressure provides the driving force for the acceleration of the expanding envelope. The UV resonance and metastable lines with envelope components, together with the central wavelengths, $v_{C}$, and the shortward edges, $v_{\max }$, of the displaced envelope lines are listed in table 3 .

Table 3. UV Resonance and Metastable Lines with Envelope Components

Ion Multiplet $\lambda_{l a b}(\AA) \quad v_{c}^{*}\left(\mathrm{~km} \mathrm{~s}^{-1}\right) \quad \mathrm{v}_{\max }^{*}\left(\mathrm{~km} \mathrm{~s}^{-1}\right)$

\begin{tabular}{|c|c|c|c|c|}
\hline C II & 1 & $\begin{array}{l}1334.532 \\
1335.705\end{array}$ & $\begin{array}{c}-710 \\
-\end{array}$ & $\begin{array}{c}-1070 \\
-\end{array}$ \\
\hline Si IV & 1 & $\begin{array}{l}1393.755 \\
1402.770\end{array}$ & $\begin{array}{l}-895 \\
-890\end{array}$ & $\begin{array}{l}-1260 \\
-1250\end{array}$ \\
\hline Si II & 2 & 1526.708 & - & -825 \\
\hline C IV & 1 & $\begin{array}{l}1548.188 \\
1550.762\end{array}$ & $\begin{array}{c}-1200 \\
-\end{array}$ & $\begin{array}{c}-2065 \\
-\end{array}$ \\
\hline Al II & 2 & 1670.787 & -735 & -970 \\
\hline AlIII & 1 & $\begin{array}{l}1854.716 \\
1862.790\end{array}$ & $\begin{array}{l}-890 \\
-830\end{array}$ & $\begin{array}{c}-1230 \\
-\end{array}$ \\
\hline $\left.\operatorname{FeIII}^{+}\right)$ & 34 & $\begin{array}{l}1895.456 \\
1914.056 \\
1926.304\end{array}$ & $\begin{array}{l}-730 \\
-790 \\
-780\end{array}$ & $\begin{array}{c}-1055 \\
-1190 \\
-\end{array}$ \\
\hline $\left.\operatorname{FeIII}^{+}\right)$ & 48 & $\begin{array}{l}2061.552 \\
2068.243\end{array}$ & $\begin{array}{l}-770: \\
-810\end{array}$ & $\begin{array}{c}-1035: \\
-\end{array}$ \\
\hline $\mathrm{Mg} I I$ & 1 & $\begin{array}{l}2795.523 \\
2802.698\end{array}$ & $\begin{array}{c}-645 \\
-\end{array}$ & -885 \\
\hline
\end{tabular}

*) Radial velocities were measured in the IUE spectra SWP 7079 and LWR 6015, obtained on 1979, Nov. 3

+) Metastable Iine 


\section{LONG-TERM VARIATIONS OF THE MASS LOSS RATE}

In contrast to the previous explanation of the period decrease by conservative mass transfer from the more to the less massive component, which is extremely unlikely to be observed due to the short thermal time scale involved in this process $(P / \dot{P} \approx 50000 \mathrm{yr})$, we suggest that the period decrease is due to loss of angular momentum carried away by the ejected matter.

A period-epoch diagram of SV Cen was established for the time between 1894 and 1981, which is based on all published photographic and photoelectric times of minimum, and has been supplemented by measurements of the Bamberg sky patrol plates and by our photoelectric observations during the recent years (Drechsel et al., 1981). First, it is obvious that the period is generally decreasing at the average large rate of $\dot{\mathrm{P}} / \mathrm{P}=-2.15 \cdot 10^{-5} \mathrm{yr}^{-1}$; and second, the rate of decrease is not constant, but highly time-dependent, with intervals of approximate constant rate of decrease, and even those with near constancy of period. Since light time effects can certainly be excluded (see, e.g., Irwin and Landolt, 1972), it is suggested that mass loss and mass exchange of the interacting binary account for the observed unsteady period decrease.

An especially efficient mechanism for accelerating the orbital revolution is ejection of matter through an external Lagrangean point. Under the assumption that the ejected material comes from the Roche lobe overflow of the less massive component through $\mathrm{L}_{1}$, is subsequently transferred to the secondary, and finally lost from the system through $\mathrm{L}_{3}$, we can apply an expression derived by Kruszewski (1966), which gives a correlation between period decrease and mass loss rate:

$$
\begin{aligned}
& \dot{\mathrm{P}} / \mathrm{P}=\frac{3}{\mu(1-\mu)} \cdot\left[\mathrm{x}\left(\mathrm{L}_{1}\right)^{2}+\frac{2}{3} \mu\left(1+\frac{\mu}{2}\right)-\mathrm{d}^{2}\right] \cdot \frac{\dot{\mathrm{M}}}{\mathrm{M}_{1}+\mathrm{M}_{2}}, \\
& \text { where } \quad=\frac{\mathrm{M}_{2}}{\mathrm{M}_{1}+\mathrm{M}_{2}} ; \mathrm{d}=\left|\mathrm{x}\left(\mathrm{L}_{3}\right)-\mathrm{x}(\mathrm{G})\right| .
\end{aligned}
$$

$\mathrm{M}_{1}$ and $\mathrm{M}_{2}$ are the individual masses of primary and secondary; $x$ denotes the rectangular Roche coordinate, i.e. d is the distance of the Lagrangean point $\mathrm{L}_{3}$ from the common center of mass, $G$.

The time derivative of the period, $\dot{p}$, was determined by differentiating the observed period-epoch correlation in sections of typically a few years. Accordingly, the mass loss 
rate was computed for the different intervals of approximate constant rate of period decrease; it is plotted in Figure 1 as a function of time between 1894 and 1981 .

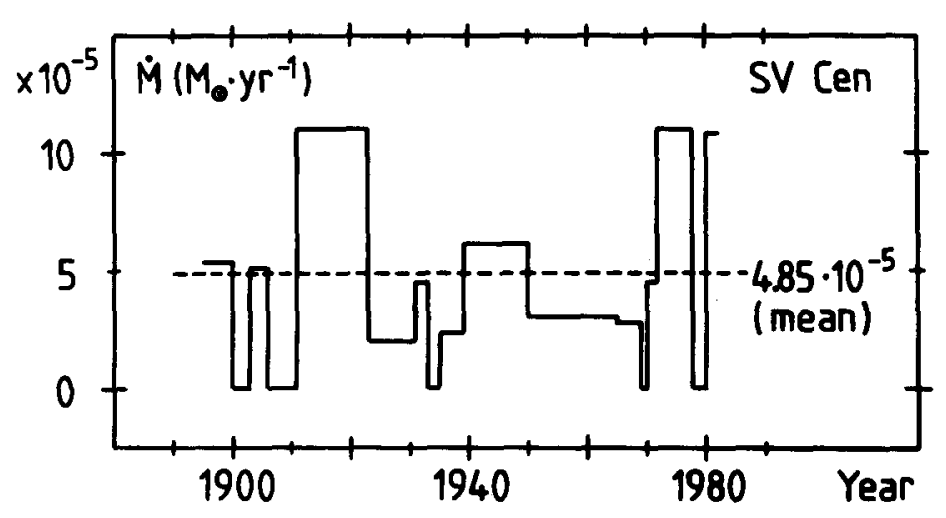

Figure 1. Mass loss rate of SV Cen for the time between 1894 and 1981 .

Major variations of the mass loss rate with time are obvious, ranging from 0 to $10^{-4} \mathrm{M}_{\odot} \cdot \mathrm{Yr}^{-1}$, with an average value of $4.85 \cdot 10^{-5} \mathrm{M}_{\odot} \mathrm{yr}^{-1}$, on a time scale of a few years.

\section{SHORT-TERM FLUCTUATIONS OF THE STELLAR WIND}

Besides the long-term variations of the mass loss rate, we found short-term fluctuations of the mass loss process from an analysis of the UV spectra obtained in late 1979 and early 1981. The striking difference between these two epochs is the appearance of several sharp absorption components superimposed on the broad envelope absorption profiles of all strong UV resonance and metastable lines in the IUE spectra taken in January 1981. As an example, the resonance doublets of C II $(1334,1335), \mathrm{Si}$ IV $(1393,1402)$, and Al III (1854, 1862 ) are shown in Figure 2. The envelope components contain at least four well-defined narrow absorption dips at nearly identical radial velocities of about $-780,-1120,-1350$, and $-1580 \mathrm{~km} \mathrm{~s}^{-1}$. 


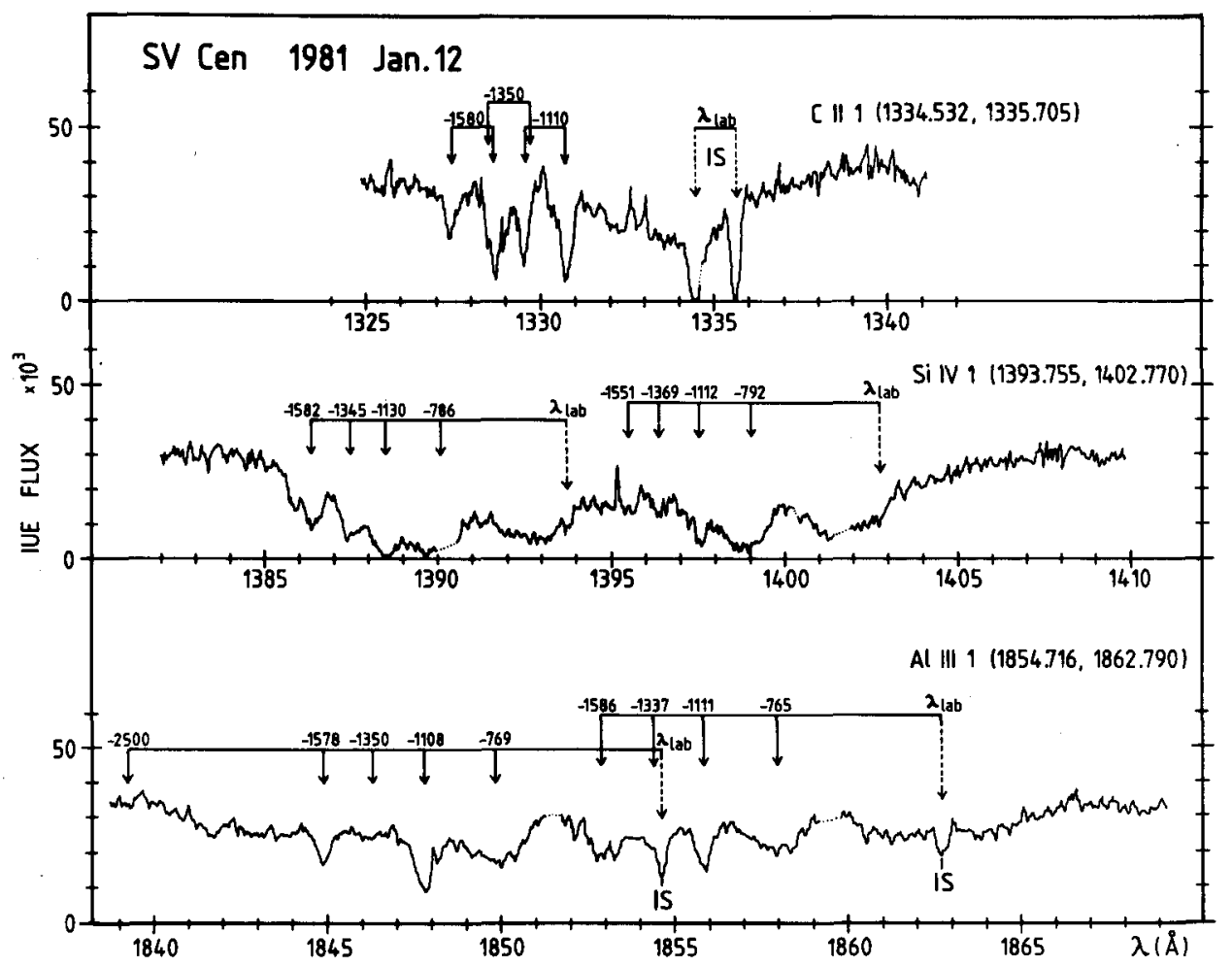

Figure 2. UV resonance line profiles of C II, Si IV, and Al III, as measured with the IUE satellite on 1981, Jan. 12 in the high resolution mode (SWP $11037)$. The envelope components contain at least four well-defined narrow absorption dips, which are indicated by arrows.

The uncontaminated envelope absorption of Si IV (1393) was used for a quantitative fit of its profile. Under the assumption of isotropic resonance scattering in a spherically symmetric expanding envelope, a theoretical representation of the general profile shape and of the narrow components could be achieved by proper adjustment of the density structure of the envelope, and for an adequate velocity law of the stellar wind. Four local condensations forming radially expanding shells can account for the well-defined absorption components. In the upper part of Figure 3, we have plotted a quantity proportional to the line optical depth which is normalized to its maximum observed value as a function of distance from the mass-losing star. The dashed line gives the best fitting velocity law in units of the observed ter- 
minal velocity of the order of $-2500 \mathrm{~km} \mathrm{~s}^{-1}$. While the high velocity part of the flow is in very good agreement with the predicted profile, the absorption strength at smaller radial velocities is systematically greater than expected. The reason might be blending with the high velocity part of the envelope absorption of the Si IV (1402) doublet transition, and possibly with some photospheric contribution. The appearance of the narrow absorption dips can therefore be attributed to the occurrence of an unsteady outflow of matter through an external Lagrangean point, giving rise to an inhomogeneous density distribution of the expanding envelope in the vicinity of the binary.

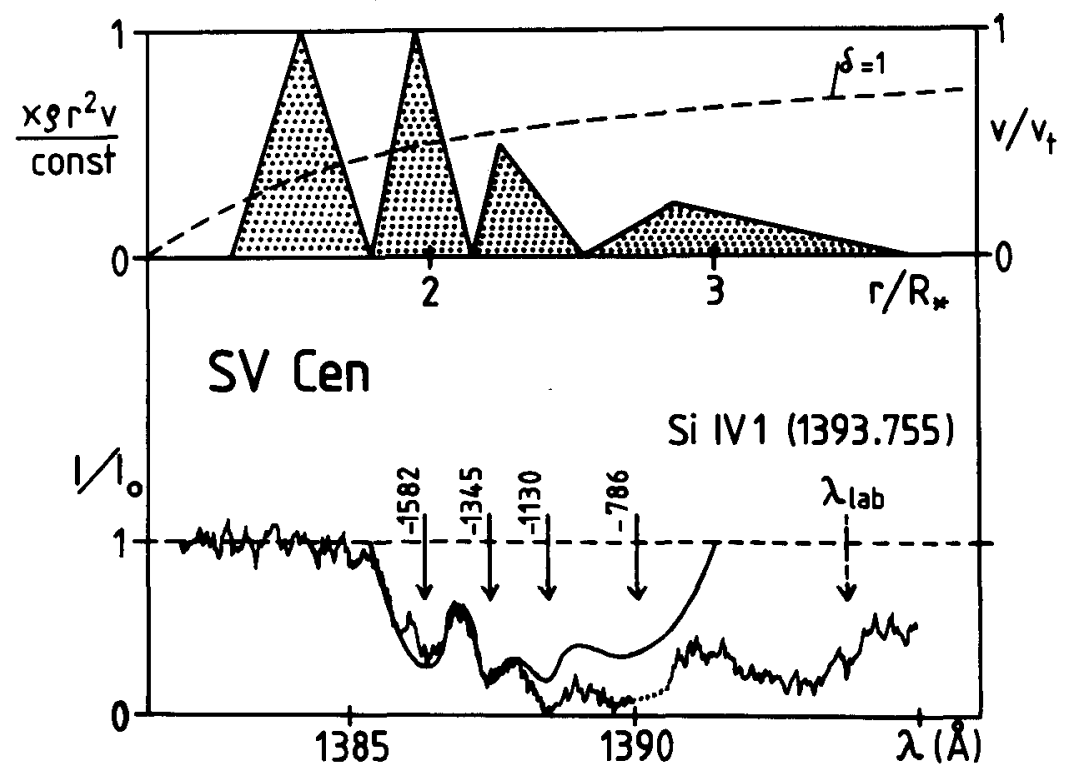

Figure 3. Measured and predicted profile of the Si IV (1393) envelope absorption line. The suggested variation of the line optical depth as a function of distance from the mass-losing star is plotted in the upper part; the dashed line indicates the best fitting velocity law.

From an integration of the velocity law

$$
v(r)=v_{t} \cdot\left(1-\frac{R_{Q}}{r}\right) \delta
$$

$\left(v_{t}=\right.$ terminal velocity; $R_{0}=$ radius of mass-losing star), 
we can estimate the time scale $\Delta t_{i, i+1}$ for the ejection of "puffs" of material producing the condensed shells $i$ and $i+1$ of the expanding envelope:

$$
\begin{aligned}
& \Delta t_{i, i+1}=\frac{1}{v_{t}} \cdot \int_{r\left(v_{i}\right)}^{r\left(v_{i+1}\right)}\left(1-\frac{R_{\varrho}}{r}\right)^{-\delta} d r, \\
& \text { with } r\left(v_{i}\right)=R_{0} /\left[1-\left(v_{i} / v_{t}\right)^{1 / \delta}\right] .
\end{aligned}
$$

The time scale comes out to be always very short, i.e. of the order of a few hours, if we assume different values for the exponent $\delta$ in the velocity law, representing high, moderate, or even low initial acceleration of the flow; for $v_{i}$ we took the radial velocities of the observed narrow absorption dips. Table 4 gives the times $\Delta t_{i}, i+1$ necessary to accelerate the stellar wind from the velocfty $v_{i}$ to $v_{i+1}$, which were obtained by numerical integration of the velocity law for a value of $\delta$ equal 1, which yielded the best theoretical representation of the Si IV (1393) envelope profile. computations for $\delta=0.5,2$, and 3 also gave typical values of $\Delta t$ equal 0.02 to 0.04 days.

Table 4. Time scale for the ejection of condensed shells

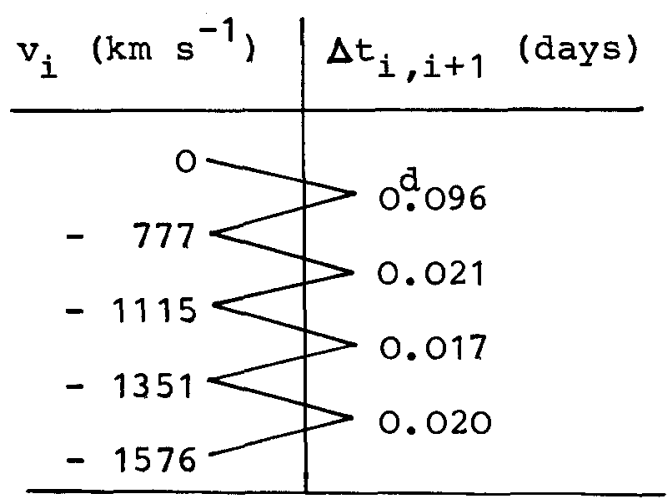

This clearly suggests that SV Cen not only shows major long-term variations of its mass loss rate on a time scale of a few years, but also exhibits a strong variability of its stellar wind within only a few hours, due to short-term fluctuations of the mass flow. 
E. PRESENCE OF A HOT SOURCE

The UV spectroscopic observations yielded the detection of a hot source in the SV Cen system. The low resolution short and long wavelength IUE spectra were combined with the photoelectric $B$ and $V$ measurements to give the absolute flux distribution in the UV and visual range.

Figure 4 shows the measured absolute intensity distribution, corrected for interstellar extinction, as filled and open triangles for the 1979 and 1981 data, respectively. The observations were obtained around quadrature, i.e. near orbital phase 0.25 . The prominent characteristic is a very strong UV excess, with the maximum of intensity distribution far beyond the IUE range.

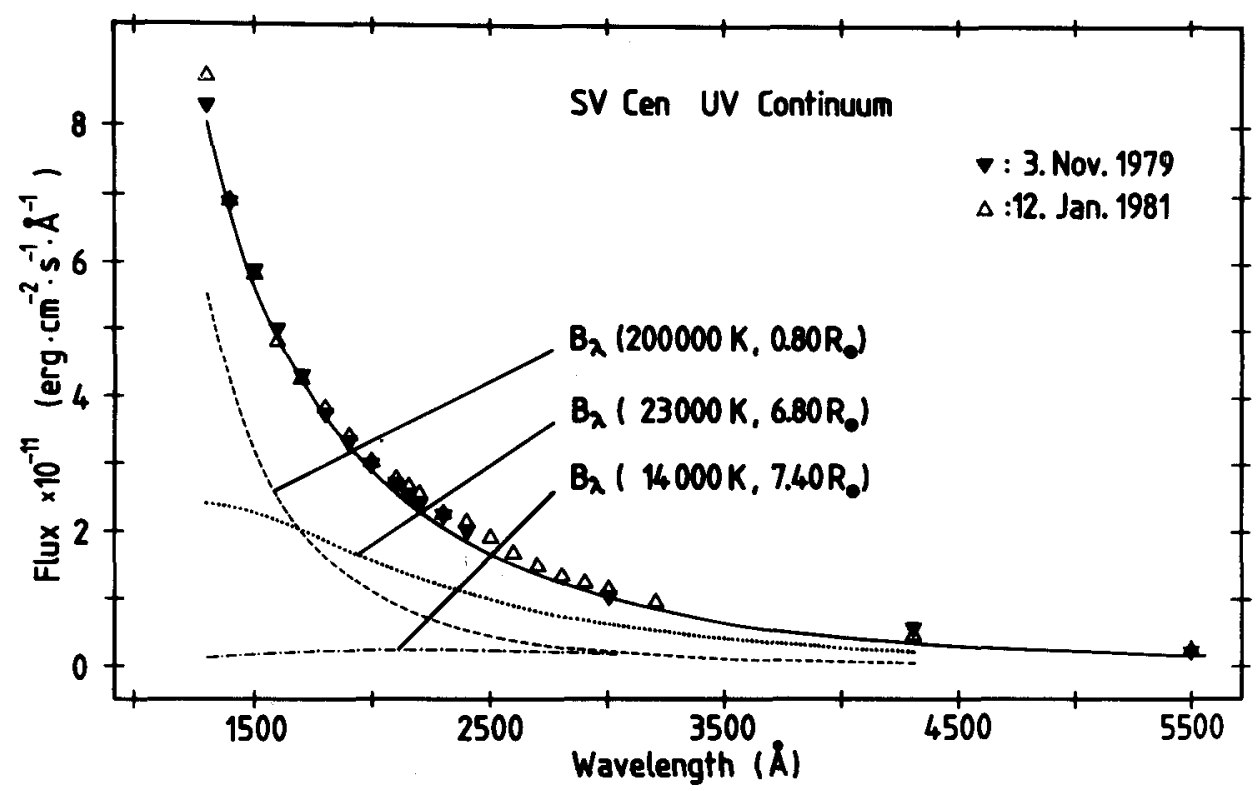

Figure 4. Absolute flux distribution in the UV and visual continuum of $\mathrm{SV}$ Cen near quadrature phase 0.25 . The UV excess is compatible with the presence of a hot ( $200000 \mathrm{~K})$ source, with an extension which is small $(\langle 1 \%)$ in comparison with the projected area of the binary components.

The measurements were interpreted by means of a three component black body fit. The dotted and dash-pointed lines represent the two stellar components, and the dashed line a 
hot area of about $200000 \mathrm{~K}$, with an extension of about 18 of the projected area of the two stars. The integrated absolute flux of all three components is plotted as solid line, and represents the measurements quite well.

\section{F. MODEL OF THE SYSTEM}

From the optical and UV measurements, the following model of SV Cen can be outlined:

The hot area can arise from dissipation of kinetic energy through shock waves connected with a hydrodynamic flow pattern originating from the Roche lobe overflow of the less massive but more luminous primary, which is travelling along the inner critical Roche surface and is approaching the outer layers of the common envelope in the equatorial region on the following side of the secondary (see Figure 5). At least part of the mass flow is leaving the system through the external $\mathrm{L}_{3}$ point, and is forming an expanding envelope which is driven by radiation pressure. From the UV spectroscopy, we can certainly exclude the conservative case of mass transfer. We suggest that the period decrease is caused by loss of angular momentum carried away by the ejected matter.

From the long-term variation of the rate of period decrease, it can be derived that the mass loss rate is variable on a time scale of a few years, and from an UV envelope line profile analysis, it is apparent that major short-term fluctuations of the stellar wind occur on a time scale of only a few hours. The reason for the variable mass loss process might be twofold:

First, it can be due to an unsteady Roche lobe overflow of the primary which is at the beginning of its post main sequence evolution; and second, it may be caused by a variation of the ratio of ejected to transferred matter. While the ejection of matter accelerates the orbital revolution, the transfer of matter from the less massive to the more massive component tends to increase the period, and both processes can conceivably combine to yield the observed behavior. 


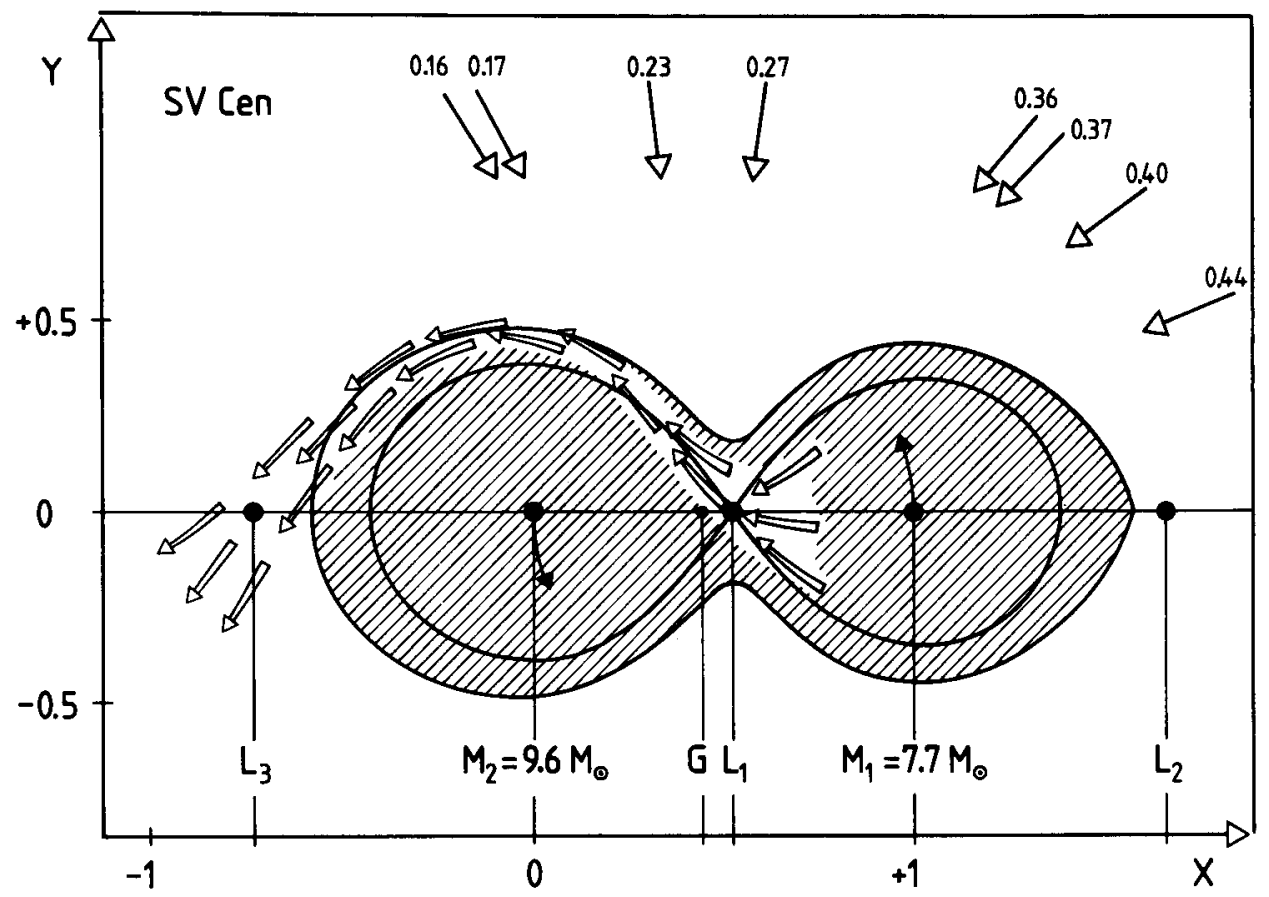

Figure 5. Model of the SV Cen system. The shaded area is the intersection of the Roche equipotential surfaces with the orbital plane. The Roche geometry corresponds to the spectroscopic mass ratio $q=1.25$. Arrows in the upper part indicate the orbital phases during which the IUE spectra were taken.

\section{REFERENCES}

Drechsel, H., Rahe, J., Wolf, B.: 1980, Proc. "Second European IUE Conference", Tübingen, 26-28 March 1980, ESA SP $-157,213$.

Drechsel, H., Rahe, J., Wargau, W., Wolf, B.: 1982, in preparation.

Irwin, J.B., Landolt, A.U.: 1972, Publ. Astron. Soc. Pacific $84,686$.

Kruszewski, A.: 1966, in "Advances in Astronomy and Astrophysics, Vol. 4", ed. Z. Kopal, Academic Press New York and London.

Wilson, R.E., Starr, T.C.: 1976, Monthly Not. Roy. Astron. Soc. 176,625 . 